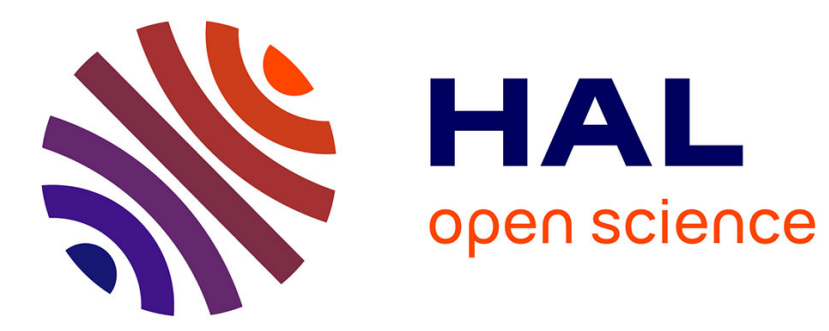

\title{
DURABILITÉ DES MATÉRIAUX PLASTIQUES TRANSPARENTS UTILISÉS ENCOUVERTURE DE CAPTEURS SOLAIRES PLANS
}

Jérome Chevalier, A. Balme, H. Sallee

\section{- To cite this version:}

Jérome Chevalier, A. Balme, H. Sallee. DURABILITÉ DES MATÉRIAUX PLASTIQUES TRANSPARENTS UTILISÉS ENCOUVERTURE DE CAPTEURS SOLAIRES PLANS. Journal de Physique Colloques, 1981, 42 (C1), pp.C1-401-C1-421. 10.1051/jphyscol:1981130 · jpa-00220680

\section{HAL Id: jpa-00220680 https://hal.science/jpa-00220680}

Submitted on 1 Jan 1981

HAL is a multi-disciplinary open access archive for the deposit and dissemination of scientific research documents, whether they are published or not. The documents may come from teaching and research institutions in France or abroad, or from public or private research centers.
L'archive ouverte pluridisciplinaire HAL, est destinée au dépôt et à la diffusion de documents scientifiques de niveau recherche, publiés ou non, émanant des établissements d'enseignement et de recherche français ou étrangers, des laboratoires publics ou privés. 
JOURNAL DE PHYSIQUE

Colloque C1, supplément au n¹, Tome 42, janvier 1981

page $1-401$

\title{
DURABILITÉ DES MATÉRIAUX PLASTIQUES TRANSPARENTS UTILISÉS EN COUVERTURE DE CAPTEURS SOLAIRES PLANS
}

\author{
J.I. Chevalier (*), A. Balme $(* *)$ et H. Sallee (*) \\ Centre Scientifique et Technique du Bâtiment, 24 rue Joseph Fourier, 38400 \\ Saint Martin d'Hères, Frànce.
}

Résumé.- Si 1'emploi de matériaux plastiques en couverture de capteur solaire présente des avantages certains, il convient néanmoins de considérer le problème de leur vieillissement en oeuvre. Une première êtape correspond à la caractérisation et à la mesure des différents agents agressifs auxquels sont soumises les couvertures transparentes. On présente les résultats d'une campagne de mesures sur les couvertures de plusieurs capteurs en fonctionnement normal et exceptionnel. Les données recueillies permettent de définir un test d'endommagement accéléré, dont l'effet est caractérisé par la mesure de I'évolution de propriétés utiles (transmission lumineuse, rësistance mécanique) dés différents matériaux considérés (polycarbonate, polyméthylmétacrylate, polyester renforcé aux fibres de verre).

Une comparaison avec l'évolution de mêmes matériaux exposés à un vieillissement naturel simple (sans absorbeur sous-jacent) devrait permettre de rechercher, une utilisation possible des connaissances đéjà acquises sur la durabilité de certains plastiques transparents utilisés dans le bâtiment en différents sites.

\begin{abstract}
The use of polymers as solar collector covers presents some real advantages, but it is necessary to consider their durability in such conditions.

The first stage consists in the identification and measurement of agressive agents which affect solar collector covers. We present the results of measurements on the covers of several solar collectors in normal and extreme working conditions.

Collected datas are used to determine an accelerated degradation test, the effect of which is characterised by useful properties measurements (solar transmittance, mechanical resistance) of the considered materials : polycarbonate, polymethylmetacrylate, glass fiber reinforced polyester.

Same measurements, done on the same materials submitted to natural wheathering (whithout absorber behind) would permit the utilisation of existing knowledge on durability of polymers in building applications.
\end{abstract}

1. Introduction.- Dans le fonctionnement thermique d'un capteur solaire plan, la couverture transparente joue un rôle essentiel, qui est de permettre l'effet de serre : elle doit pour cela transmettre la plus gran-
(*) Cellule Solaire du CSTB
(**) Division "Physique des Matériaux" 
de partie possible du rayonnement solaire incident, et absorber lou réfléchir dans le cas des couvertures transparentes dites sélectives) les rayons infrarouges provenant de 1 'absorbeur. Elle doit aussi confiner une lame d'air au-dessus de l'absorbeur, de façon à limiter les échanges convectifs. De façon contradictoire, la couverture transparente est donc par l'effet de serre à l'origine de l'échauffement de l'absorbeur, et par la réémission des rayons infrarouges le siège des pertes thermiques les plus importantes du capteur solaire.

Ses propriétés essentielles seront donc ses caractéristiques optiques et sa tenue mécanique. Elles devront présenter un facteur de transmission énergétique élevé de 0,3 à 2,2 microns, c'est-à-dire sur 1 'étendue du spectre solaire, et une opacité totale aux infrarouges lointains, de 4 à $30 \mu$, c'est-à-dire aux longueurs d'onde d'émission du corps noir correspondant à des températures de $100^{\circ} \mathrm{C}$ à $-20^{\circ} \mathrm{C}$ (en tenant compte de la largeur des spectres).

Elles devront d'autre part présenter une bonne résistance aux chocs et aux sollicitations mécaniques, qui peuvent être dues à des variations de pression ou aux simples dilatations différentielles. Le verre est généralement choisi pour les couvertures de capteurs solaires à cause de ses propriétés optiques. Néanmoins sa tenue aux chocs ne peut être assurée en fonctionnement gu'au prix d'une épaisseur élevée (4, parfois $6 \mathrm{~mm}$ ) ou de traitements particuliers (trempe). Il reste deux inconvénients majeurs à l'emploi du verre : son poids et les risques cle casse accidentelle en transport, en manutention ou lors de la pose.

C'est pourquoi de nombreux fabricants de capteurs solaires s'orientent vers l'emploi de matières plastiques, moins lourdes et moins fragiles. Mais si le verre peut être considéré comme un matértau de référence en vieillissement, l'emploi de matériaux polymériques nécessite une étude sérieuse de leur durabilité, et c'est ce sujet que nous allons aborder. 2. Les matériaux utilisés et les connaissances actuelles sur leur durabilité. - Trois matières plastiques rencontrent actuellement des emplois en couverture de capteur solaire :

- le polymétylmétacrylate (PMMA),

- le polycarbonate (PC),

- le polyester renforcé aux fibres de verre (PRV).

La figure 1 donne pour des produits commerciaux disponibles en France et aux Etats Unis les propriétés essentielles à titre indicatif /1,2,3/. La figure 2 montre les spectres types de transmission lumineuse globale (directe plus diffuse) obtenus pour chaque matêriau dans la gamme UV, visible et proches.

Ces trois matériaux sont connus et employés dans certaines applications bâtiment depuis déjà de nombreuses années. 
Le PNMA est un polymẽre thermoplastique caractérisé par une transparence normale élevée, une bonne résistance aux intempéries, et une faible absorption d'eau. Il est par contre facilement rayable, a une tenue en température faible, présente un comportement au feu médiocre $\left(M_{3}-M_{4}\right)$ et sa résistance aux chocs est assez faible, car il s'agit d'un matériau mécaniquement fragile.

\begin{tabular}{|c|c|c|c|c|c|c|c|c|}
\hline Nateriau & $\begin{array}{l}\text { Ppais. } \\
\text { produit } \\
\operatorname{mm}\end{array}$ & $\begin{array}{l}\text { Facteur de } \\
\text { transmis- } \\
\text { sion global } \\
\text { "solaire" } \\
\text { (b) }\end{array}$ & $\begin{array}{c}\text { Temperature } \\
\text { maximale } \\
\text { d'emploi }^{\circ}\left({ }^{B} \mathrm{C}\right)\end{array}$ & $\begin{array}{l}\text { Coefficient } \\
\text { de dilata- } \\
\text { tion } \\
\mathrm{cm} / \mathrm{cm} /{ }^{\circ} \mathrm{c} \\
\left(\mathrm{x} 10^{-5}\right)\end{array}$ & Densite & $\begin{array}{l}\text { Module } \\
\text { elastique } \\
\text { da.y/mg? } \\
\left(x \quad 10^{5}\right)\end{array}$ & $\begin{array}{l}\text { Charge de } \\
\text { rupturg } \\
\text { da:y/mm? }\end{array}$ & $\begin{array}{l}\text { Absorption } \\
\text { d'eav } \\
\left(\begin{array}{lll}1 & \text { en } 24 & h\end{array}\right)\end{array}$ \\
\hline $\begin{array}{r}\text { Polycartonate } \\
\text { (PC) }\end{array}$ & 3,2 & $\begin{array}{l}82-89(2) \\
8.4(3)\end{array}$ & $\begin{array}{l}110-130(2) \\
100-130\end{array}$ & $\begin{array}{r}6,7(2) \\
(1)\end{array}$ & 1,2 & $2,3^{(2)}$ & $6,5(2)$ & $0,15^{(1)}$ \\
\hline $\begin{array}{l}\text { Polyméthyl- } \\
\text { metacrylate } \\
\text { (1Pnit) }\end{array}$ & 3,2 & $\begin{array}{l}99^{(2)} \\
89-92(1)\end{array}$ & $\begin{array}{l}30-90(?) \\
60-03^{(1)}\end{array}$ & $\begin{array}{l}3,8(2) \\
5-9(1)\end{array}$ & 1,17 & $3,1(2)$ & $\begin{array}{l}7,2^{(2)} \\
,(3)\end{array}$ & $0,3-0,4^{(1)}$ \\
\hline $\begin{array}{l}\text { Polycser } \\
\text { renforce aux } \\
\text { fibres Je } \\
\text { rerre (fRV } \\
\text { 2s: de fintes } \\
\text { de verre }\end{array}$ & $\begin{array}{l}1,3 \\
3,2\end{array}$ & $\begin{array}{l}77-90^{(2)} \\
86^{(3)}\end{array}$ & $95(2)$ & $3,2-1(2)$ & $1,3-1,5$ & $\begin{array}{l}7,6^{(2)} \\
7^{(3)}\end{array}$ & $\begin{array}{l}10-12^{(2)} \\
11,5^{(3)}\end{array}$ & $\begin{array}{l}0,12^{(1)} \\
0,16\end{array}$ \\
\hline Verre blanc & 3 & $85-9 i^{(2)}$ & $200(2)$ & $0,9-1,5(2)$ & 2,5 & $72,3^{(2)}$ & $\begin{array}{l}2,8^{(2)} \\
6,9^{(2)} \\
(\text { trerape) }\end{array}$ & \\
\hline
\end{tabular}

Fig.1.- Caractéristiques des différents matériaux plastiques utilisés en couverture de capteurs solaires.

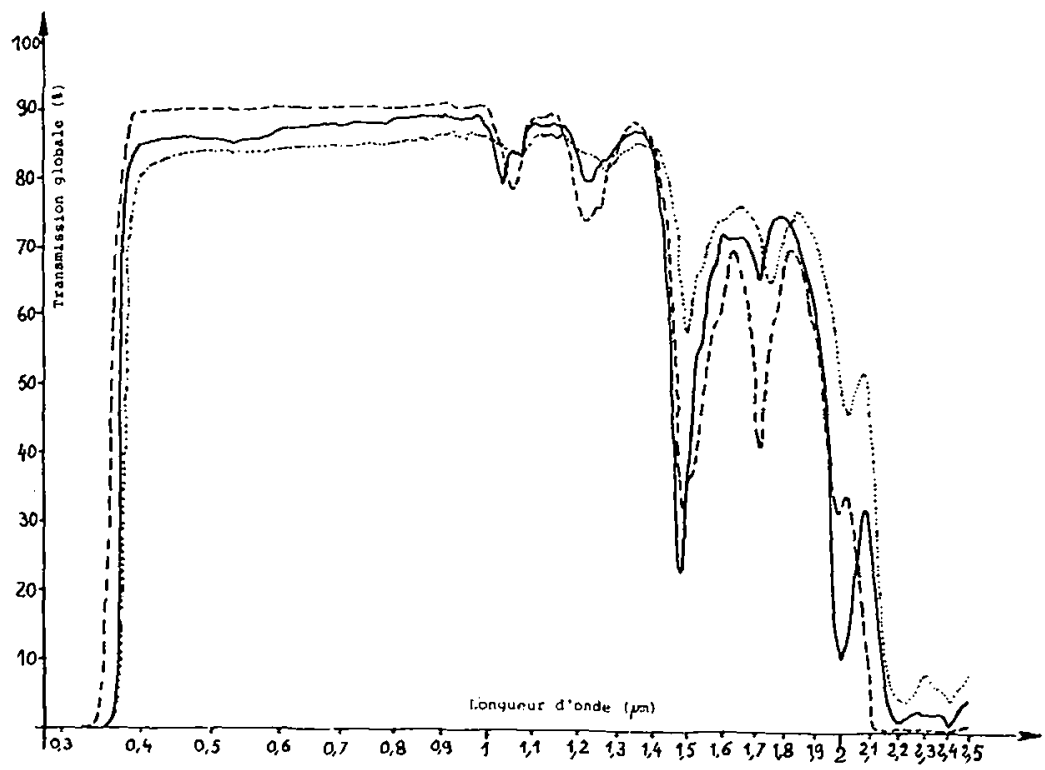

Fig.2.- spectres type de transmission globale pour trois matériaux plastiques transparents.

ester : polycarbonate poly-

Le polycarbonate, lui aussi thermoplastique, a une meilleure tenue en température. Il est connu pour son bon comportement mécanique et notam- 
ment sa grande résistance au choc. Les qualités de transparence sont satisfaisantes. Sa durabilité est bonne. Cependant sa dureté superficielle relativement faible le rend sensible à toute dégradation de surface, et son comportement au feu est comparable à celui du PMMA. Le PRV est un matériau composite obtenu par adjonction de fibres de verre, sous forme de mât de verre, ou de façon dispersée dans une résine polyester, polymère thermodurcissable. Ses propriétés mécaniques sont élevées. Elles dépendent beaucoup du type et de la concentration de charges renforçantes; elles sont meilleures dans les produits stratifiés (mât de verre) que dans les produits chargés. Les matériaux courants comportent en général entre 15 et 25 \% de fibres de verre.

La transparence du PRV est bonne, si l'on considère la transmission globale, car une bonne partie de la lumière transmise est diffusée. Mais les résines standard conférent au PRV une tenue limitée au vieillissement et aux intempéries. Le comportement au feu peut varier de $\mathrm{M}_{2}$ à $\mathrm{M}_{4}$ suivant le type de résine.

Dans le domaine du bãtiment, ces matériaux ont fait l'objet d'une part d'études de vieillissement, et d'autre part d'expositions naturelles contrôlées. On dispose donc de connaissances partielles sur la durabilité de ces matériaux dans le domaine du bâtiment.

Rappelons d'abord les différents facteurs climatiques responsables du vieillissement naturel /4/:

- le rayonnement ultraviolet : il provient du rayonnement solaire. La partie UV non absorbée par l'atmosphère s'étend de 290 à $400 \mathrm{~nm}$. Ces radiations représentant environ $5 \%$ du spectre solaire sont potentiellement agressives. En effet les photons de cette gamme de longueur d'ondes ont des énergies qui correspondent à celles de certaines liaisons chimiques, qui peuvent être de ce fait rompues /5, 6/. L'absorption de ces photons par les matériaux organiques ne se traduit pas obligatoirement pax une dégradation photochimique pure. La dégradation d'un polymère soumis au rayonnement en présence d'oxygène suit un processus complexe de réactions en châ̂nes /27/ dont l'amorçage peut être d'origine photochimique ou thermique (consécutivement à l'absorption des photons par des pigments ou additifs chromophores). La manifestation physique la plus évidente de cette dégradation est une coloration au matériau souvent associée à une fragilisation.

- l'eau : sous forme liquide (pluie, condensation) ou sous forme gazeuse (humidité de l'air) présente trois modes d'actions :

- chimique : c'est l'hydrolyse de certaines liaisons moléculaixes, la création de sites photosensibles, ou la favorisation de certaines dégradations biologiques.

- physique : ce sont par exemple les phénomènes d'adsorption ou d'absorption suivis d'un séchage avec extraction d'un additif. 
- mécanique : ce sont les actions de lessivage, d'érosion superficielle. - l'oxygène et l'ozone : la concentration en oxygène est sensiblement constante. Son action, favorisée par la présence de radiations ou une élévation de température se situe au niveau des doubles liaisons moléculaires résiduelles. L'ozone, bien que présent en faible quantité dans l'atmosphère normale $\left(\leqslant 0,03 \mathrm{mg} / \mathrm{m}^{3}\right)$ est particulièrement agressif pour les polymères insaturếs.

- la température, dans le cas d'utilisations classiques dans le battiment, ne peut s'élever que très rarement aux environs de $70^{\circ} \mathrm{C}$. Ce facteur climatique a donc exceptionnellement à lui-seul un effet destructeur. Cependant il pilote les cinétiques des dégradations engendrées par d'autres facteurs : hydrolyse, photolyse, oxydation.

- les polluants atmosphériques : et parmi eux l'anhydride sulfureux, l'anhydride carbonique, les oxydes d'azote, mais aussi les poussières et suies ont une incidence déterminante sur l'aspect, et sur la transmission lumineuse (par action chimique, ou par dépôt de salissures). Les connaissances actuelles sur la durabilité des trois matériaux cités face à ces agents agressifs peuvent être résumêes de la façon suivante : - Le PMMA est classé parmi les polymēres les plus stables face à 1'action de la lumière /7, 11/. Il sera néanmoins légèrement sensible aux rayons ultraviolets, qui en présence d'oxygène peuvent donner lieu à des ruptures de chaines surtout si la température est élevée. Le poids moléculaire va donc diminuer au cours du temps, mais sans pour autant conduire à des variations sensibles de la transmission lumineuse et des propriétés mécaniques. Si une légère coloration apparầt parfois, on peut penser à la présence d'impuretés ou d'additifs instables /8/. Les évolutions peuvent être suivies par la mesure de la transmission lumineuse et de la résistance au choc. L'êtude de la viscosité en solution permet d'accéder au poids moléculaire.

- Le polycarbonate, qui présente une bonne stabilité photochimique est sensible au rayonnement ultraviolet, qui peut provoquer une légère coloration. Certains auteurs /9/ mentionnent également 1 apparition de microfissures, qui sont attribuées à l'effet conjugué des rayons ultraviolets et des cyclages thermiques. Comme pour le PMMA on assiste à des scissions de chaInes et une diminution du poids moléculaire qui peut se traduire par de fortes variations des caractéristiques mécaniques. On peut suivre le vieillissement du PC par mesure de transmission lumineuse (et on constatera surtout une augmentation du pourcentage de rayonnement diffus plus qu'une diminution du facteur global) et par mesure de résistance au choc.

- Le vieilissement naturel des PRV se caractérise par deux manifestations essentielles /10/:

- une dégradation superficielle qui est la résultante de plusieurs com- 
posantes, en particulier l'hydrolyse de la couche superficielle de résine, l'érosion mécanique, ces phénomènes étant accélérés par le développement des craquelures et la résurgence des fibres de verre.

- une dégradation de la liaison entre les deux composants fibres et résine et ceci même dans la masse du matériau.

L'état de dégradation superficielle, ainsi que le jaunissement de la résine dans la masse peuvent être appréciés par une mesure de transmission lumineuse globale, alors que la mesure du module de rigidité en torsion permet d'apprécier la dégradation de l'adhérence fibres-résine, qui influe directement sur l'ensemble des propriétés mécaniques et physiques.

3. Les couvertures transparentes de capteurs solaires: nécessité d'une étude spécifique de durabilité.- Nous venons de voir que les matériaux utilisés dans la fabrication des capteurs solaires sont pour la plupart connus pour d'autres applications dans le domaine du bâtiment.

On dispose donc d'informations sur leur comportement face au vieillissement. Des tests de durabilité ont été mis au point pour certains d'entre eux. Ces connaissances sont bien sûr utiles à l'étude de la durabilité de ces matériaux lorsqu'ils entrent dans la fabrication d'un capteur solaire, mais on ne peut transposer directement ces tests et ces informations à cette nouvelle application car les conditions auxquelles les matériaux sont soumis sont différentes.

Nous travaillons donc à la mise au point de tests spécifiques de-durabilité des matériaux utilisés en capteurs solaires. La ligne directrice pour la mise au point d'un tel test procède du schéma classique /12/ : après avoir identifié le matériau étudiê, on doit préciser d'abord ses conditions de travail, puis appréhender les facteurs de dégradation, mesurer leurs effets et rechercher les indices de dégradation. Ces indices sont suivis en dégradation artificielle, et on recherche une corrélation avec des mesures en vieillissement naturel.

Les références dans ce domaine sont peu nombreuses hormis les documents réalisês directement par des laboratoires industriels, traitant du produit qu'ils commercialisent /13, 14/.Signalons que le NBS a lancé aux Etats Unis un vaste programme avec un objectif voisin de celui du CSTB $/ 15 /$.

Dans notre étude, la définition des produits servant aux essais est importante. Les PMMA, étant donnée leur bonne durabilité dans des conditions normales, seront des produits standards. Les PC seront eux des nuances stabilisées UV, et les PRV devront être de conception spéciale, étant donnée leur sensibilité aux rayons UV : on choisira des produits présentant en surface une couche de résine PMMA ou un film de protection, par exemple en polyfluorure de vinyl. 
Pour la détermination des nouvelles conditions de travail, nous avons réalisé une plate-forme d'exposition. Cette plate-forme, déjà décrite précedemment /16/ comprend dix capteurs couvrant pratiquement l'éventail des materiels existants, et notamment les differents matériaux utilises couramment pour chaque composant. Chaque capteur est en série sur un circuit hydraulique independant permettant la cixculation du fluide par effet de thermosiphon.

Un ballon en acier inoxydable non isole thermiquement sert a la fois de vase d'expansion et de deperditeur thermique. Les constituants de chaque circuit sont adaptés au matériau de l'absorbeur pour éviter tout problème de corrosion par couple galvanique.

Les capteurs sont orientés plein sud, et leur inclinaison peut être réglée suivant la saison, pour rechercher les conditions optimales de captation.

Chaque composant de capteur est equipe d'un thermocouple. Le rayonnement global et la température extérieure sont également mesurés. Ces données sont relevées toutes les heures par une centrale de données couplée a une imprimante. A cet ensemble, nous avons adjoint un microordinateur permettant de piloter la centrale. La souplesse de l'ensemble permet a l'aide de quelques programmes, de traiter les données de différentes manières : (plusieurs graphes en parallèle avec le rayonnement ou la temperature extérieure, ou histogrammes de températures pour chaque composant sur plusieurs mois).

Les histogrammes des figures 3 et 4 montrent que, sans être aussisollicitées en température que les absorbeurs /17/, les couvertures transparentes sont néanmoins soumises à un régime plus sevère que dans les autres applications bâtiment des matériaux transparents (bardages, vitrages). Leur temperature peut occasionnellement atteindre $100^{\circ} \mathrm{C}$ sur $1 a$ face exposée à l'absorbeur.

Les differences saisonnières sont importantes. Les histogrammes présentent, à la belle saison, des maximums relatifs dans la zone des températures les plus elevées.

on ne distingue pas de différence sensible suivant le type de revétement d'absorbeur : un revêtement sélectif s'échauffe plus mais réémet moins, et les effets thermiques sont identiques au niveau de la couverture. on a pu remarquer egalement un gradient important entre la face intérieure de la couverture et la face extérieure. Cette différence est plus grande dans le cas des matières plastiques, moins conductrices que le verre. Elle est par contre faible pour la vitre interieure d'un double vitrage mais les temperatures atteintes sont plus elevees ( jusqu'a $\left.130^{\circ} \mathrm{C}\right)$. 


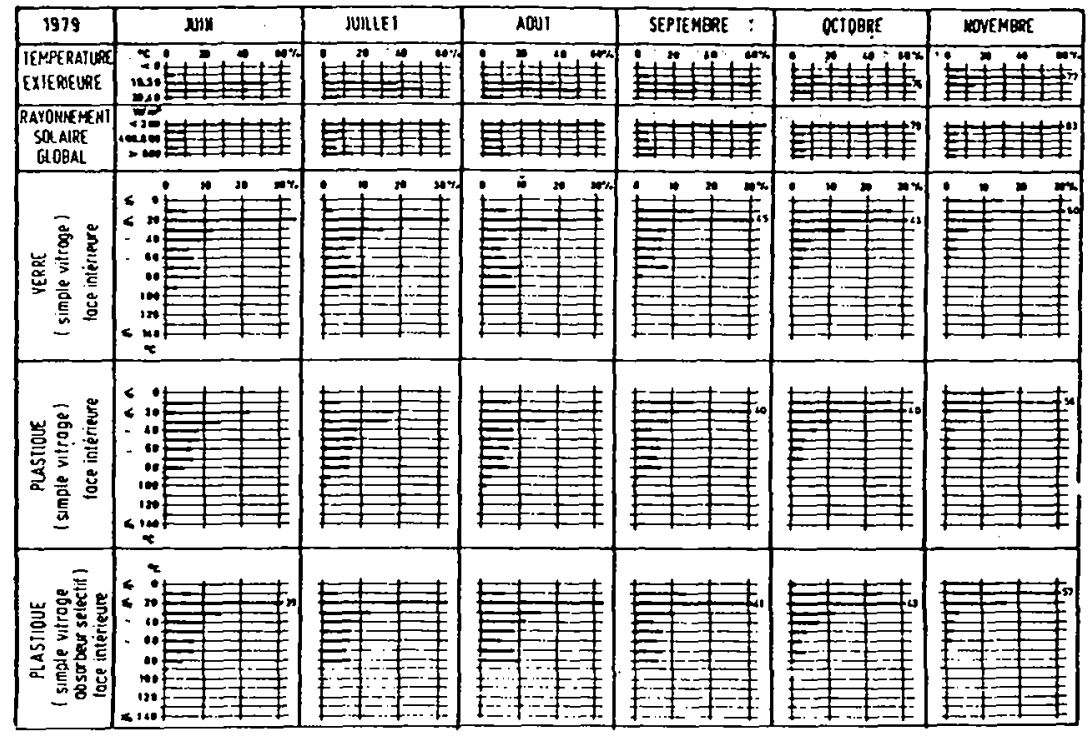

Fig.3.- Histogramme de temperatures des couvertures de capteurs solaires (exemple: la longueur de la barre a $" \leqslant 50^{\circ} \mathrm{C} "$ correspond au pourcentage de temps passé entre 40 et $50^{\circ} \mathrm{Cl}$.

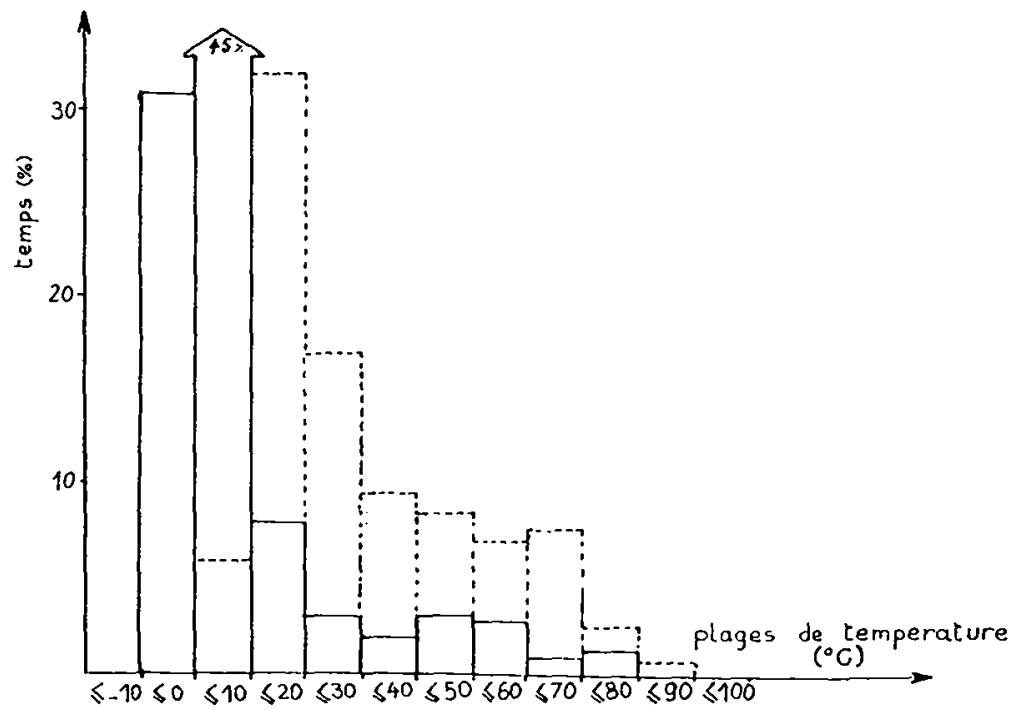

Fig.4.- Comparaison des histogrammes de températures en juillet et en janvier 1980 pour une couverture de capteur en PMMA.

- : janvier $1980 ;----$ : juillet 1979 ;

Couverture PMMA (température face intérieure)

Capteur classique. 
En stagnation (Fig.5) des temperatures approchant $120^{\circ} \mathrm{C}$ ont eté enregistrées sur la face intérieure de couvertures plastiques.

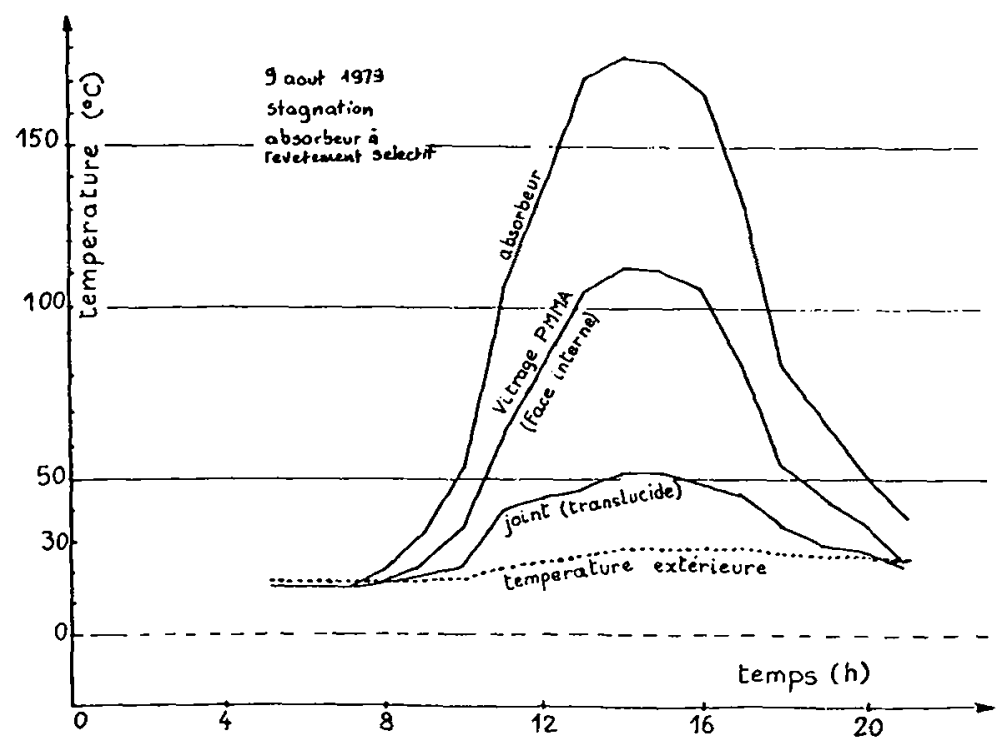

Fig.5.- Température obtenues dans deux capteurs en stagnation le 9 aout 1977 (couverture en PMMA).

Les différentes valeurs de temperatures auxquelles peuvent être soumises les couvertures transparentes sont en bon accord avec les résultats experrimentaux du NBS/18/ et légèrement supérieures à celles calculées au CSTB dans un cas legerement different /19/ (vitre devant une paroi absorbante exposée au soleil. Ce cas peut être rapproche de la stagnation sèche).

4. La méthode d'etude de la durabilité et les premiers resultats.- Nous avons défini trois matériaux susceptibles d'être utilisés comme couverture de capteurs solaires. Nous avons constate que les conditions de travail dans ce cas sont originales : les temperatures maximales sont élevées, et le cyclage thermique journalier a une grande amplitude. Les facteurs de degradation seront donc essentiellement le rayonnement ultraviolet et la température qui va jouer un rôle important. Dans un premier temps, nous avons recherché les indices de dégradation pour les propriétés fonctionnelles des matériaux : nous avons suivi pour cela la transmission énergétique et les caractéristiques mécaniques.

Le facteur de transmission solatre global est calculé à partir du spectre de transmission globale (directe + diffuse) obtenue au spectrophotomètre équipè d'une sphère d'intégration, dans une gamme qui s'étend de 0,3 à 2,5 microns. 
Le suivi des caractéristiques mecaniques est plus délicat : la même méthode ne peut en effet $s$ 'appliquer à la fois à un polymère thermoplastique et à un matériau composite.

Pour les thermoplastiques, nous avons utilisé des mesures de perforation. Ce test dêjà utilisé pour suivre le comportement mécanique du PVC et du polycarbonate, qui n'est pas encore généralisé au niveau des laboratoires, est cependant bien approprié à l'etude du comportement d'un matériau à un choc accidentel. On utilise une machine de traction hydraulique dont la vitesse maximale est de $12 \mathrm{~m} / \mathrm{s}$. L'éprouvette est un carré de $30 \mathrm{~mm}$ de côté, et le perforateur est une tige de $4 \mathrm{~mm}$ de diamètre. On mesure et on enregistre la force de pénétration en fonction du deplacement relatif éprouvette-perforateur effectué à vitesse constante.

On apprécie d'une part l'aspect de l'éprouvette. On note deux types de comportement : un comportement fragile, et un comportement ductile. Pour un materiau donne, dans un etat de vieillissement donné, la transition ductile-fragile a une position fixe dans un diagramme température-vitesse de sollicitation. Au cours du vieillissement, cette transition peut se déplacer.

D'autre part, on peut mesurer l'énergie fournie a l'éprouvette pour la perforer, en intégrant la courbe de rupture.

Pour les polyesters renforcés cet essai n'aurait aucun sens, du fait de la présence des fibres de verre.

Nous avons alors envisagé l'évolution du module de rigidité en torsion, mesure au pendule de torsion.

Le pendule de torsion qui a été mis au point au laboratoire /26/ est de type inversé.

La fréquence d'oscillation du système pendulaire, (éprouvette et volant d'inertie) est de l'ordxe de 1 hertz.

Le pendule est entretenu par un ensemble bobines-aimants asservi par un système optique dont les photodiodes servent aussi à la mesure de la periode.

Pour un échantillon de section rectangulaire le module de rigidité en torsion est inversement proportionnel au carré de la periode du mouvement oscillatoire.

La précision sur la mesure du paramètre $\mathrm{G}$ est essentiellement fonction de la précision de mesure des paramètres géometriques de l'éprouvette et en particulier de son épaisseur étant donné que la période peut être mesurée à l'aide des périphériques électroniques précédemment cités, avec une précision inférieure a $5 \times 10^{-4}$.

Le paramètre $G$ est fonction également, et c'est ce qui nous intéresse, de l'état d'endommagement du matériau. 
Dans le cas d'une utilisation en couverture de capteurs solaires, le "vieillissement naturel" est difficile à définir. En l'absence de prélèvements réels, nous avons utilisé des échantillons mis en place sur le capteur Arlequin. Ce capteur, installe sur la plate-forme décrite précédemment comporte une couverture constituée déchantillons de différents materiaux couvrant la gamme du marché ( $\left.{ }^{1}\right)$.

Nous avons aussi suivi deux matériaux qui équipaient deux autres capteurs de la plate-forme, et dont nous avons pu obtenir des échantillons témoins $\left({ }^{2}\right)$.

Le but de l'étude est de définir un test de durabilité significatif, grâce auquel une prévision de duré de vie dans l'utilisation en couverture de capteur solaire sera possible pour la materiau envisagé. D'après les développements précedents, il apparait que l'endommagement doit faire intervenir à la fois le rayonnement UV et la temperature. Décrivons le dispositif dans lequel ont été réalisés les essais exploratoires.

Nous avons utilisé un dégradeur ultraviolet, équipé d'une lampe au xénon de 6000 watts, dans une enceinte ventilée dont la température peut être régulée. L'intensité de la lampe est également régulée. On dispose donc d'une source d'uV connue.

Les échantillons ont été disposés en couverture de mini-capteurs réalisés en contre-plaqué, dont l'absorbeur est une plaque metallique peinte en noir. L'étanchéité est assurée par un joint silicone (Fig.6). D'autre part les filtres anticaloriques ayant été remplacés par des filtres en verre au borosilicate, le spectre du rayonnement s'étend de $290 \mu$ à $2500 \mu$, et présente une bonne similitude avec le rayonnement solaire à midi l'été. L'énergie mesurée est de $1050 \mathrm{w} / \mathrm{m}^{2}$. La température d'air est maintenue à $30^{\circ} \mathrm{C}$ environ, mais les echantillons sont soumis du fait du montage à $60^{\circ} \mathrm{C}\left( \pm 2^{\circ} \mathrm{C}\right)$ sur la face exposée en UV, et à $80^{\circ} \mathrm{C}\left( \pm 2^{\circ} \mathrm{C}\right)$ sur la face opposee, ce qui reconstitue grossièrement les conditions sévères auxquelles ces matériaux peuvent être soumis dans un capteur solaire. Les échantillons PM 12, PM 23, PC 22, PC 33, PR Il et PR 21 ont été sou-

( $\left.{ }^{1}\right)$ Il s'agit de :

- deux PMMA référencés

- deux PRV réfêrencés

- deux PC référencés

\section{PM 12 (extrudē)}

PM 23 (coulé)

PR 11 (dopé au PMMA)

PR 21 (recouvert de PVF)

PC 22 (d'origine differente)

PC 32 (stabilisés UV)

$\left({ }^{2}\right)$ Il s'agit d'un PMMA référencé PM 24 et d'un PC xéférencé PC 42 non stabilisé UV. 
mis pour la phase exploratoire a 400 heures d'exposition à ce test que l'on appellera "UV + tempërature".

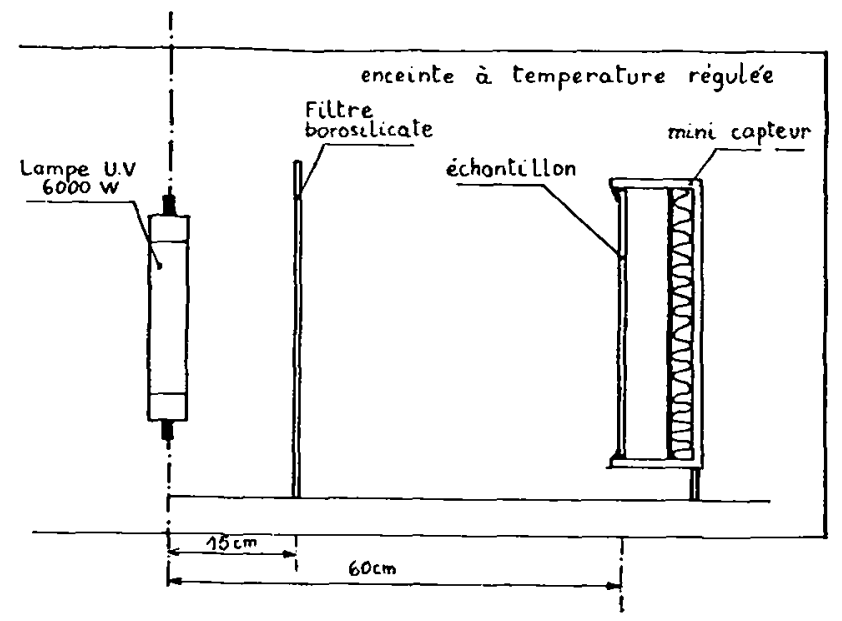

Fig.6.- Schéma de disposition des échantillons en endommagement artificiel (UV + température).

Nous allons maintenant commenter les premiers résultats obtenus.

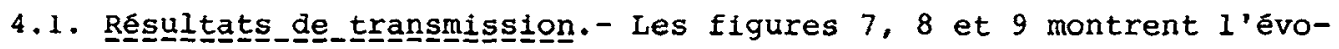
lution des spectres de transmission globale entre 0,3 et $2,5 \mu$ après 1 an en capteur Arlequin, et après 400 heures d'"UV + temperature", pour PM 12, PC 22 et PR 21.

On constate que les spectres presentent peu de variation sensible sur toute leur etendue. Les pics d'absorption dans le proche infrarouge sont stables même si parfois leur intensité augmente, et le vieillissement ne se manifeste pas par des variations de forme dans ce domaine, ou par un deplacement de la limite de transmission.

Lorsque des differences apparaissent, c'est dans le domaine UV visible, par une augmentation de l'absorption vers les courtes Iongueurs d'onde. Cet effet conduit d'une part a une baisse de transmission et d'autre part a une absorption de photons de longueur d'onde eventuellement dangereuse pour le matériau.

La figure 10 donne les valeurs du facteur de transmission solaire globale. Ce facteur est le résultat d'une prise en compte du spectre solaire incident : celui-ci est decoupe en plusieurs bandes d'égale énergie et la moyenne des pourcentages de transmission dans chaque bande est appelée facteur de transmission solaire globale $/ 30 /$.

Les variations après 6 mois ou 1 an d'exposition sur le capteur Arlequin ne sont significatives, que si on les rapproche de l'evolution des spectres dans le rayonnement UV et visible desfigures 7,8 et 9 . 


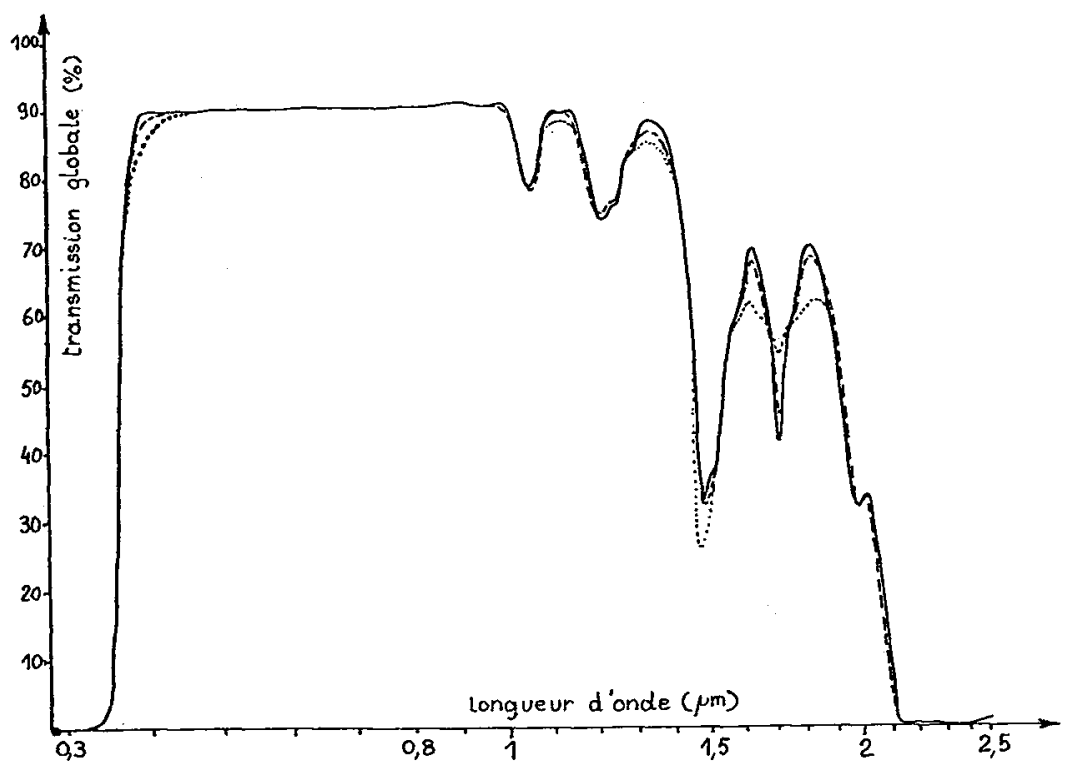

Fig.7.- Evolution des spectres de transmission globale en cours de vieillissement (PMMA).

Polymethylmetacrylate PM 12, - neuf ; - :- : 1 an Arlequin; ...: : $400 \mathrm{~h}$ UV + température.

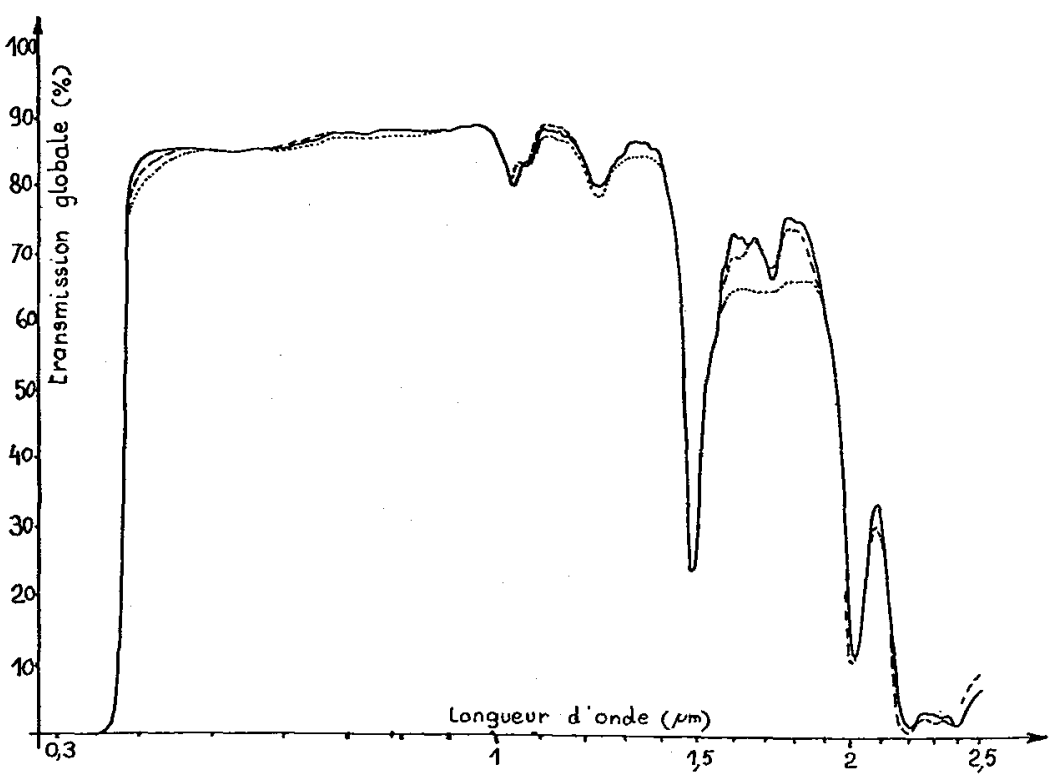

Fig.8.- Evolution des spectres de transmission globale en cours de vieillissement (PC). Polycarbonate PC 22, - : neuf ; --- 1 an Arlequin; ....4 40Oh ov + température. 


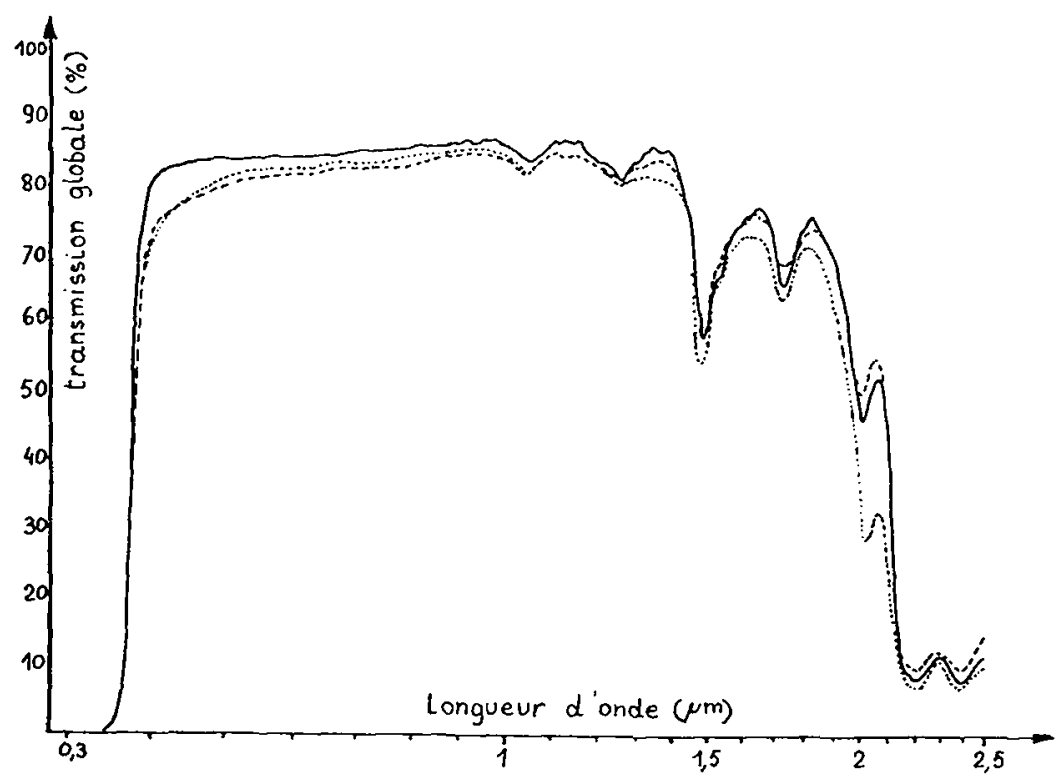

Fig.9.- Evolution des spectres de transmission globale en cours de vieillissement (PRV). Polyester renforce verre PR 21, - : neuf; -.- : 1 an Arlequin ; $\ldots$ : $: 400 \mathrm{~h} \mathrm{UV}+$ température.

\begin{tabular}{|c|c|c|c|c|}
\hline \multirow{3}{*}{$\begin{array}{l}\text { Matériau } \\
\text { PC } 22\end{array}$} & \multirow{3}{*}{$\begin{array}{l}\text { Témoin } \\
0,76\end{array}$} & \multicolumn{2}{|c|}{ Exposition Arlequin } & \multirow{3}{*}{ 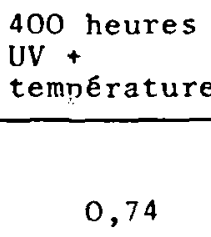 } \\
\hline & & 6 mois & 1 an & \\
\hline & & 0,76 & $0,76^{\prime}$ & \\
\hline PC 32 & 0,75 & & 0,76 & 0,74 \\
\hline PM 12 & 0,81 & 0,80 & 0,80 & 0,77 \\
\hline PM 23 & 0,78 & 0,78 & 0,79 & 0,78 \\
\hline PR 11 & 0,71 & 0,70 & 0,70 & 0,63 \\
\hline PR 21 & 0,76 & 0,75 & 0,74 & 0,74 \\
\hline
\end{tabular}

Fig.10.- Pourcentage de transmission globale des differents materiaux après six mois et un an en service sur Arlequin, et après $400 \mathrm{~h}$ d'endommagement artificiel UV + temperature.

On peut comparer ces mesures après des temps courts d'exposition a des résultats d'observation en vieillissement naturel simple. Des échantillons de PMMA ont montré une stabilité totale en transparence après 10 ans d'exposition à Champs sur Marne $/ 24 /$, ce qui est également le cas 
de nuances de polycarbonate traitées anti-UV exposé à Champs sur Marne et à Grenoble $/ 28 /$, pendant deux ans.

Par contre, des échantilions de PRV recouverts d'un film de polyfluorure de vinyl (PVF) (ce qui est le cas du produit PR 2l) ont montré après 6 ans d'exposition dans le Nord de la France une baisse sensible de transmission lumineuse, estimé a 15 \& a $0,5 \mu / 29 /$.

De l'observation visuelle des échantillons prélevés sur le capteur Arlequin, il faut retenir une légere modification d'aspect des polycarbonates (dépoli en surface) qui ne se traduit pas par une modification de la transmission globale mais par une baisse du pourcentage de transmission directe, et un jaunissement sensible des échantilions de PRV. L'endommagement "UV + temperature" a donné Iieu lui, à des évolutions plus significatives, surtout pour le PR ll, du facteur de transmission. L'aspect des échantillons est lui aussi altêré, le jaunissement étant surtout sensible sur les PC et les PRV.

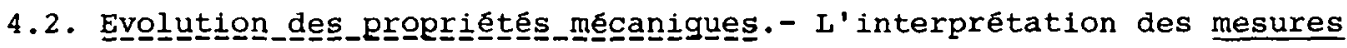
de perforation doit tenir compte de l'observation du type de rupture, et de la courbe effort-déformation enregistrée lors de l'essai. La figure 11 montre d'une part 1 'aspect des éprouvettes donnant lieu à une rupture fragile et ductile, et d'autre part les courbes enregistrées lors d'une perforation sur un PMMA et sur un polycarbonate.

RUPTURE DUCTILE DU POLYCARBONATE
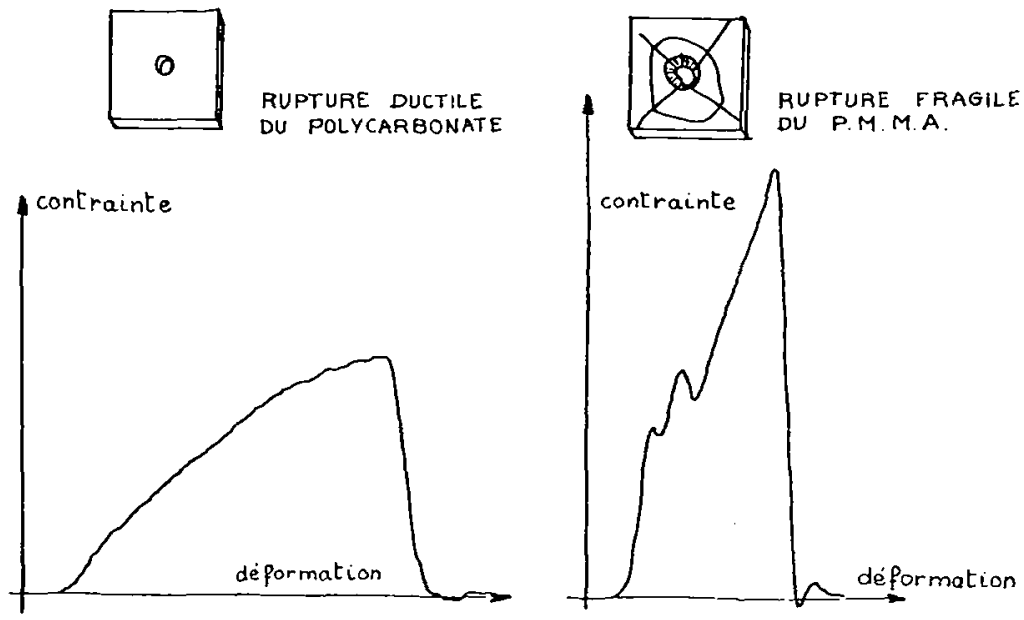

Fig.11.- Courbes-type et état de l'éprouvette lors d'essais de perforation a $3 \mathrm{~m} / \mathrm{s}$ sur des PMMA et des $\mathrm{PC}$.

Sur la figure 12 apparaissent les charges à la rupture mesurées sur les $\mathrm{PC}$ et les PMMA, et les types de fractures obtenues. 


\begin{tabular}{|c|c|c|c|c|c|}
\hline \multicolumn{2}{|c|}{$\begin{array}{l}\text { Natériau } \\
\text { Epaisseur }\end{array}$} & Témoin & 1 an Arlequin & $\begin{array}{l}400 \text { heures } \\
\text { UV + } \\
\text { température }\end{array}$ & \multirow[t]{2}{*}{1 an capteur } \\
\hline PC 22 & $2 \mathrm{~mm}$ & $162 \pm 8$ (D) & $172 \pm 7$ (D) & $159 \pm 4$ (D) & \\
\hline PC 32 & $2 \mathrm{~mm}$ & $154 \pm 10(2)$ & $153 \pm 8$ (D) & $165 \pm 4(2)$ & \\
\hline PC 42 & $2 \mathrm{~mm}$ & $149 \pm 9(D)$ & & & $\begin{array}{l}40 \pm 6 \text { face } \\
(F) \text { avant } \\
164 \pm 32 \text { face } \\
(D) \quad \text { arrière }\end{array}$ \\
\hline PM 12 & $\begin{array}{l}2 \mathrm{~mm} \\
3 \mathrm{~mm}\end{array}$ & $\begin{array}{l}57 \pm 3 \\
\text { (F) } 92 \pm 13\end{array}$ & $38 \pm 9$ (F) & $70 \pm 13(F)$ & \\
\hline PM 23 & $3 \mathrm{~mm}$ & (F) $85 \pm 14$ & $71 \pm 9$ (F) & $46 \pm 3$ (F) & \\
\hline PM 24 & $4 \mathrm{mmi}$ & $(F)^{132 \pm 10}$ & & & $138 \pm 11(F)$ \\
\hline
\end{tabular}

Fig.12. - Charge de rupture en perforation (en daN) our le PC (essais à $-30^{\circ} \mathrm{C}$ ) et le PMMA (essais à $+23^{\circ} \mathrm{C}$ ).

(D) rupture ductile; (F) rupture fragile.

Les PMMA sont déjà fragiles à l'état neuf à la température ambiante. Des mesures a $70^{\circ} \mathrm{C}$ sur PM 12 ont montré un comportement du même type. Néanmoins, on peut noter une baisse sensible de la charge à la rupture aussi bien après vieillissement naturel et endommagement artificiel. La forme de la courbe type obtenue appelle une remarque : la présence d'un ou plusieurs maximums de contrainte avant la montée qui conduit a la rupture est due au fait que dans le PMMA, I'énergie de propagation d'une fissure est du même ordre que celle qui est nécessaire pour l'initier /21/. La première fissure crêée lors de l'essai de perforation, et qui donne lieu à la relaxation de contrainte, n'est pas celle qui conduira à la rupture.

pour cette raison aussi il faut éviter de comparer des essais réalisés sur des éprouvettes d'épaisseursdifférentes.

Le produit PM 24 a été utilise sur un capteur de la plate-forme pendant 1 an. Est alors apparu sur la face interne (soumise au plus hautes températures) un réseau assez dense de petites fissures. Nous avons attribue cette dégradation au résultat de contraintes mécaniques de dilatation, la fixation de la couverture n'étant pas satisfaisante. La force engendrée par la dilatation est en effet de nature a creer en surface une contrainte suffisante pour initier des fissures car l'énergie de création des fissures diminue si la temperature augmente $/ 20 /$. On voit sur la figure 12 que ces fissures ne donnent pas lieu a une diminution des propriétés mécaniques, même si la sollicitation est appli- 
qué z sur la face opposée aux fissures, la aussi l'énergie élevée de propagation des fissures explique ce résultat. La figure 13 montre que le spectre UV-visible $n$ 'est affecté que très faiblement.

Les polycarbonates ont été testés a $-30^{\circ} \mathrm{C}$. Les fractures observées sont ductiles elles le seront donc a fortiori à des températures plus élevées.

On constate une stabilité des charges de rupture après les divers endommagements. On peut noter néanmoins que certains échantillons présentent une fracture qui reste ductile, mais dont les lèvres sont le siège de phénomènes locaux de type fragile, après 400 heures d'exposition UV + tempērature.

Enfin le produit PC 42, ductile a l'état neuf, est tiré d'un profil extrude tubulaire dont la face avant est la couverture transparente, et la face arrière sert de coffre pour l'isolant du capteur.

Un comportement fragile a été décelé à $-30^{\circ} \mathrm{C}$ pour un échantillon préleve en face avant (comportement qui a pu être confirmé a la temperature ambiante), alors que la face arrière reste ductile.

Cette fragilisation due à l'effet des UV et de la température ne s'accompagne que d'une légère modification du spectre UV-visible (Fig.13).

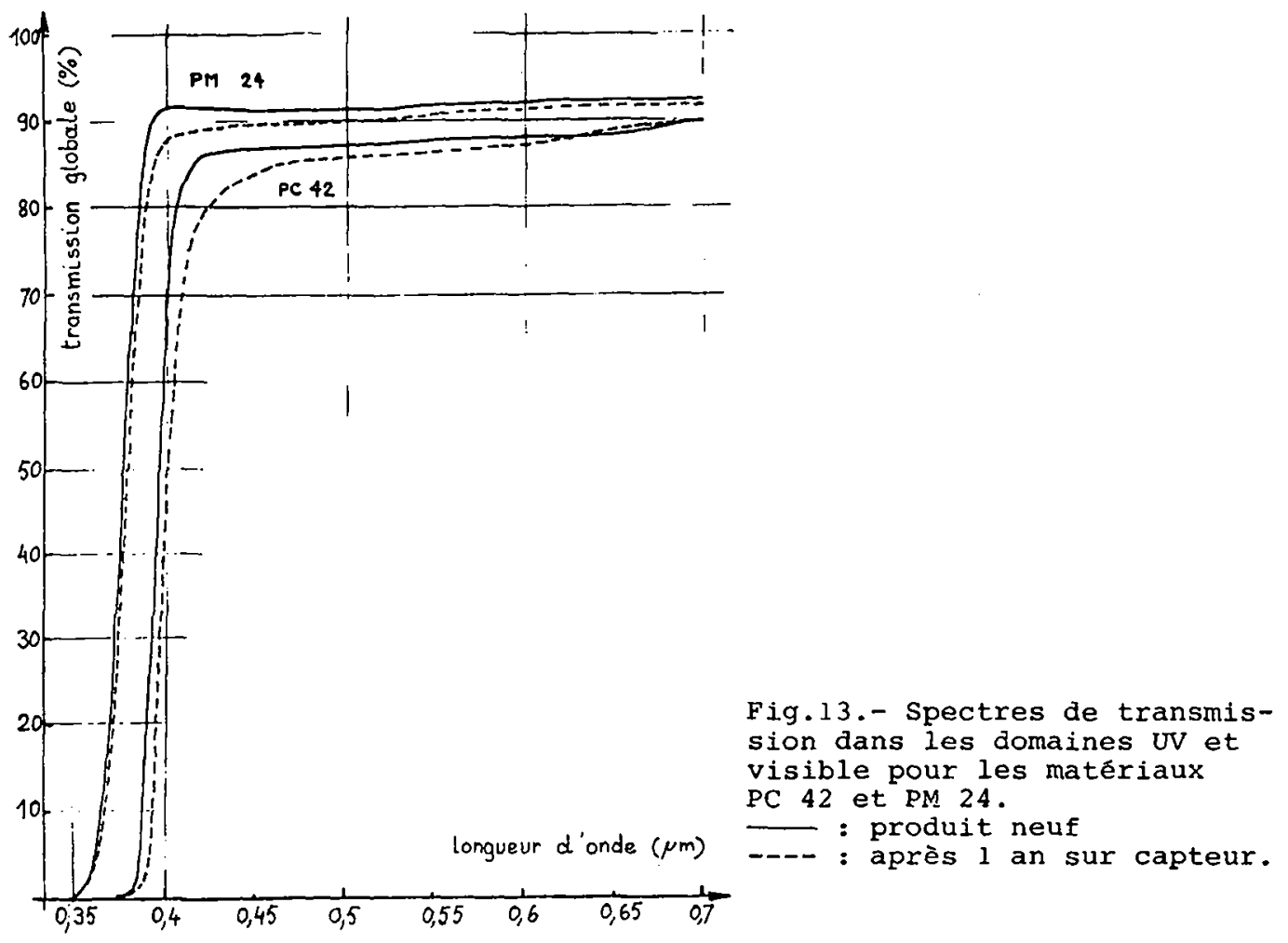


Si une exposition de ce produit a l'endommagement UV + temperrature donnait lieu a une fragilisation, nous aurions une confirmation de la validité de notre test. Cet endommagement est actuellement en cours. 4.3. Mesures_de_modules. - La figure 14 donne les mesures de modules realisees au pendule de torsion sur les deux PRV. Le seul commentaire, que ces résultats inspirent, consiste à prendre note d'une grande stabilité de la tenue mécanique des PRV testés, même après des endommagements qui modifient leur aspect et leur facteur de transmission.

\begin{tabular}{|l|c|c|l|}
\hline Matériau & Témoin & 1 an Arlequin & $\begin{array}{l}400 \text { heures } \\
\text { UV }+ \text { rérature } \\
\text { temperature }\end{array}$ \\
\hline PR 11 & $157 \pm 6$ & $145 \pm 3$ & $165 \pm 1$ \\
\hline PR 21 & $151 \pm 4$ & $185 \pm 5$ & $153 \pm 2$ \\
\hline
\end{tabular}

* Cette valeur, peu coherente avec la mesure sur le produit témoin, a éte obtenue sur un matériau d'epaisseur plus faible $(0,8 \mathrm{~mm})$ que celle du témoin $(1,3)$.

Fig.14.- Mesures de modules de torsion sur des échantillons de PRV (en $\mathrm{daN} / \mathrm{mm}^{2}$ ).

5. Conclusion. - L'étude de la durabilite des materiaux plastiques rigides pour leur utilisation en couvertures transparentes de capteur solaire doit faire l'objet d'un travail spécifique. L'endommagement UV + température doit, pour constituer un test significatif faire l'objet d'essais supplémentaires sur de bons et de mauvais produits, connus pour leur comportement en oeuvre.

On constate néanmoins que les premiers produits qui lui ont été soumis ont vu leur caractéristiques fonctionnelles (facteur de transmission solaire globale et propriétés mécaniques) modifiées sensiblement dans les mêmes conditions qu'après un vieillissement en oeuvre.

Les exemples fournis par les matériaux prêlevés sur capteurs réels montrent qu'il faut être prudent avec les modifications d'aspect (qui n'entrainent pas toujous une modification des caractëristiques fonctionnelles) et que des mesures à la fols de transmission lumineuse et de propriêtés mécaniques doivent être faites pour donner un avis sur le matériau.

Enfin pour les produits necessitant une formulation ou une structure speciale de protection aux rayons UV, l'identification préalable est essentielle. 


\section{Bibliographie}

/1/ "Recommandations pour la réalisation d'une installation solaire dans l'habitat"

AFEDES (1978)

/2/ NBS (communication privée)

Solar Technology Group (1979)

/3/ "Optimal material selection for flat plate solar energy collectors utilizing commercially available materials"

Ratzel, A.C., Bannerot, R.B.,

Nuclear, Solar and Process heat Transfer, St Louis, (1976) SYMPOSIUM

/4/ "Les agents du viellilssement naturel"

CSTB, Division "Physique des Matériaux"

REEF (réédition) à paraitre (1980)

/5/ "Simulation de l'action solaire"

Ammar, C., Longuet, M. ,

RILEM, Commission Liants Résineux, GT4 : Durabilitê (1974)

/6/ "Le vieillissement des matières plastiques"

Verdu, J.,

Rapport C.E.M.P., (1973)

17/ "Plastiques, comportement à long terme"

Verdu, J.,

"Techniques de l'Ingénieur" A 3150

/8/ "Use of plastics in solar energy application"

Blaga, A.,

Sol. Energy, 21 (1978)

19/ "Degradation of polycarbonate sheet on outdoor exposure" Yamasaki, R.S., Blaga, A.,

"Materials and Structure" RILEM 10 (1977)

/10/ "Contribution à l'etude du vieillissement naturel des résines polyester renforcées de fibres de verre"

Cope, R.,

Cahiers du CSTB, $\mathrm{N}^{\circ} 1426$, 1ivraison 177 (1977)

/11/ "Effect of outdoor aging on acrylic sieet"

Rainhart, L.G., Schimmel, W.P.

Sol. Energy 17 (1975) 259

112/ "La durabilitê des matières plastiques dans leurs emplois de la captation solaire"

Eurin, Ph., Chevalier, J.L., Balme, A., Sallee, H. Journées d'étude du GPCP (1979)

/13/ "Wheatherability of fiber glass solar collector covers" White, J.S.,

Polym. News $\underline{3}$ (1977) 
/14/ "Acrylic plastic glazing : properties, characteristics and engineering data"

Burkhardt, W.C.,

Ashrae Trans. 82 (1976)

/15/ "The NBS solar collector durability/reliability programm" Streed, F.R., Waksman, D., Document NBS

/16/ Cahiers du CSTB, $n^{\circ} 1563$, livraison 198, (1979) pp 301-306

/17/ "Etudes sur la durabilité des composants solaires" Chevalier, J.L., C.R. Activité 1979, à paraitre dans les Cahiers du CSTB (1980)

/18/ "Experimental verification of a standard test procedure for solar collectors" Hill, J.E., Jenkins, J.P., Jones, D.E., NBS Report, Building Science Series 117

/19/ "Temperatures maximales atteintes au soleil par des parois de construction" Croiset, M., Berthier, J., Cahiers du CSTB, $n^{\circ} 467$, livraison $66,(1962)$

/20/ "Fracture du polymethacrylate de méthyle" Boisserie, C., Mémoire CNAM, (1974)

/21/ "Propagation d'une fissure Energie Specifique de surface de fracture dans les polymères" Schirrer, R., Mémoire Scientifique de la Revue de Metallurgie, (1980)

/22/ "Outdoor performance of plastics" Rossiter, W.J., NBS Report NBSIR 73.146, (1980)

123/ "Solar Energy Systems, Swvery of Material Performance" Skoda, L.F., Masters, L.W., NBS Report MBSIR 77.1314 (1977)

/24/ "Vieillissement naturel des matières plastiques utilisables dans la construction"

Rechner, L.,

Cahiers du CSTB, $n^{\circ} 1453$, (1977)

/25/ "Etude du comportement mécanique du polycarbonate" Roux, G., Cahiers du CSTB, $\mathrm{n}^{\circ} 1568,(1979)$

126/ "La mesure des caractéristiques mécaniques des matériaux Chevalier, J.L., Cope, R., Peguin, P., Roux, G., Cahiers du CSTB, $\mathrm{n}^{\circ} 1314$, (1975)

/27/ "Vieillissement des polymères empirisme ou science ?" Lemaire et al. RGCP $n^{\circ} \frac{{ }^{\circ}}{593,}$ (1979) 
/28/ "Le point de 1'année 1979"

Division "Physique des Matériaux" (1980)

Document CSTB

/29/ "Méthodes d'étude du vieillissement des plastiques" Eurin, Ph., Journée AFEDES sur les matériaux transparents pour capteurs solaires, Grenoble, (1978)

/30/ CEN-G. (Communication privée)

DMG/Laboratoire d'Etude des Matériaux Minces, (1980) 